\title{
Bayesian Estimation of Measurement Error Models with Longitudinal Data
}

\author{
Dewang LI \\ School of Mathematics and Big data \\ University of Huizhou \\ Huizhou, Guangdong Province, China \\ e-mail: ldwldw1976@126.com
}

\author{
Meilan QIU \\ School of Mathematics and Big data \\ University of Huizhou \\ Huizhou, Guangdong Province, China \\ e-mail: qml_1981@126.com
}

\begin{abstract}
In this paper, Bayesian inferences for semiparametric measurement error models (MEs) for longitudinal data are investigated. A semiparametric Bayesian approach combining the stick-breaking prior and the Gibbs sampler together with the Metropolis-Hastings algorithm is developed for simulating observations from the posterior distributions and producing the joint Bayesian estimates of unknow parameters and measurement error. We obtain Bayesian estimations of parameters and covariates subject to MEs. Two simulation studies are presented to illustrate our proposed methodologies.
\end{abstract}

Keywords-Bayesian; measurement error; longitudinal data

\section{INTRODUCTION}

Longitudinal data frequently occur in medical follow-up studies and analysis of environmental data, and various methods for analysis these data have been proposed. Lin and Carroll [1]. Measurement error data are often encountered in many fields, including engineering, economics, physics, biology, biomedical science and epidemiology. Statistical inference methods for various parametric MEs have been well established over the past several decades. A systematic survey on this research topic was given and many applications of measurement error data were presented by Fuller (1987) [2] and Carroll, Ruppert, Stefanski and Crainiceanu (2006) [3]. Measurements with errors have been used to study in partially linear models by many scholars, such as Wang, Lin, Gutierrez and Carroll (1998) [4]; Liang, Härdel and Carroll (1999) [5]; Ma and Carroll (2006) [6]; Liang , Wang and Carroll (2007) [7]; Pan, Zeng and Lin (2008) [8]. In this paper, a hybrid algorithm is proposed to generate observations required for a Bayesian inference from the posterior distributions of parameters and covariates subject to ME which is combined with the distribution of Beta and Gamma or Beta and Beta by combining the stick-breaking prior and the Gibbs sampler together with the Metropolis-Hastings algorithm.

\section{MEASUREMENT ERROR MODELS}

For $i=1, \ldots, n$, suppose that $y_{i}$ is the observed outcome variable, $Z_{i}$ be a $r \times 1$ vector of the unobserved covariate variables, and $\mathrm{U}_{i}$ be a $p \times 1$ vector of the observed covariate variables for the $i$ th individual. Let
$Z_{i}=\left(X_{i}^{T}, U_{i}^{\mathrm{T}}\right)^{T}$, we assume that $y_{i}$ 's are conditionally independent of each other. For longitudinal data, we consider the generalized linear measurement error models of the following structure

$$
p\left(y_{i} \mid X_{i}, \psi\right)=\exp \left\{\frac{y_{i} \tau_{i}-d\left(\tau_{i}\right)}{\psi}+c\left(y_{i}, \psi\right)\right\}
$$

Where

$$
\gamma_{i}=E\left(y_{i} \mid X_{i}\right)=\dot{d}\left(\tau_{i}\right)
$$

$\mu_{i}=\operatorname{Var}\left(y_{i} \mid X_{i}\right)=\zeta \ddot{d}\left(\tau_{i}\right), \psi$ is a scale parameter, $\mathrm{d}($.$) and \mathrm{c}(\cdot, \cdot)$ are specific differentiable functions, $\dot{d}\left(\alpha_{i}\right)=\partial d\left(\tau_{i}\right) / \partial \tau_{i}$ and $\ddot{d}\left(\tau_{i}\right)=\partial^{2} d\left(\tau_{i}\right) / \partial \tau_{i}^{2}$. The conditional mean $\gamma_{i}$ is satisfied the equation

$$
\lambda_{i}=g\left(\gamma_{i}\right)=Z_{i}^{T} \alpha_{z}+U_{i}^{T} \alpha_{V}=X_{i}^{T} \alpha .
$$

Where $\mathrm{g}(\cdot)$ is a monotonic differentiable link function, $\beta=\left(\beta_{z}^{T}, \beta_{V}^{T}\right)^{T}$ is a $(r+p) \times 1$ vector of unknown regression coefficients. As in [9], in this paper, we assume that the true covariate $Z_{i}$ are measured $m$ times for individual $i$ with additive errors, and $\mathrm{Y}_{i} Z_{i}$ are errors free. That is, we cannot observe $Z_{i}$ but we can observe $W_{i j}$ for $j=1, \ldots, m$ with

$$
\mathrm{W}_{\mathrm{ij}}=Z_{i}+\theta_{i j}
$$

Where the MEs $\theta_{i j}$ 's are assumed to follow an unknown distribution, and are independent of $Z_{i}$. As in Lachos (2010) [10], we suppose the Dirichlet process (DP) mixture model to specify the distribution of $\theta_{i j}$.

To discriminate specification of the covariate ME model, we define a true covariate model for $Z_{\mathrm{ki}}(k=1, \ldots, r)$ is 


$$
Z_{\mathrm{ki}}=\beta_{k 0}+\beta_{k V}^{T} U_{i}+\xi_{k i}, \quad \xi_{k i} \sim N\left(0, \sigma_{z}^{2}\right)
$$

Where $\rho_{k 0}$ is an intercept, $\rho_{k v}=\left(\rho_{k 1}, \ldots, \rho_{k p}\right)^{T}$ is a $p \times 1$ vector of unknown regression parameters. Denote $Y=\left\{y_{1}, \ldots, y_{n}\right\}, Z=\left\{Z_{1}, \ldots, Z_{n}\right\}, U=\left\{U_{1}, \ldots, U_{n}\right\}$, $\theta=\left\{\theta_{1}, \ldots, \theta_{n}\right\} \quad$ and $W=\left\{W_{1}, \ldots, W_{n}\right\} \quad$ in which $Z_{i}=\left(z_{1 i}, \ldots, z_{r i}\right)^{T} \quad, \quad \theta_{i}=\left(\theta_{i 1}, \ldots, \theta_{i m}\right) \quad$ and $W_{i}=\left\{W_{i 1}, \ldots, W_{i m}\right\}$ for $i=1, \ldots, n$. Denote $\varepsilon_{y}=\{\alpha, \psi\}$, $\varepsilon_{\beta}=\left\{\beta_{10}, \ldots, \beta_{r 0}, \beta_{1 V}, \ldots \beta_{r V}, \sigma_{z}^{2}\right\}, \varepsilon_{\theta}$ are parameters of equation (3) and $\varepsilon=\left\{\varepsilon_{y}, \varepsilon_{\beta}, \varepsilon_{\theta}\right\}$. The joint probability density function for $\{Y, W, \theta, Z\}$ is expressed as

$$
\begin{gathered}
P(Y, W, \theta, Z \mid U, \varepsilon)= \\
\prod_{i=1}^{n}\left\{p\left(y_{i} \mid Z_{i}, U_{i} ; \varepsilon_{y}\right) p\left(W_{i} \mid Z_{i} ; \varepsilon_{\theta}\right) p\left(Z_{i} \mid U_{i} ; \varepsilon_{\beta}\right)\right\} .
\end{gathered}
$$

We assume the following priors for parameters $\alpha, \quad \psi, \beta_{k}=\left(\beta_{k 0}, \beta_{k v}^{T}\right)^{T}$ for $k=1, \ldots, r \quad$ and $\sigma_{z}^{2}$ are given by

$$
\begin{gathered}
\alpha \psi, \alpha^{0}, H_{\alpha}^{0} \sim N_{r+p}\left(\alpha^{0}, \psi^{-1} H_{\alpha}^{0}\right), \\
\psi^{-1} \mid b_{1}, b_{2} \sim \Gamma\left(b_{1}, b_{2}\right), \\
\beta_{k} \mid \beta_{k}^{0}, H_{\beta k}^{0} \sim N_{p+1}\left(\beta_{k}^{0}, \psi^{-1} H_{\beta k}^{0}\right), \\
\sigma_{z}^{-2} \mid d_{1}, d_{2} \sim \Gamma\left(d_{1}, d_{2}\right) .
\end{gathered}
$$

Where $b_{1}, b_{2}, \alpha^{0}, H_{\alpha}^{0}, \beta_{k}^{0}, H_{\beta k}^{0}, d_{1}$ and $d_{2}$ are hyperparameters whose values are supposed to be given by the prior information. According to the above presented joint probability density function and priors, a Bayesian approach is considered to make statistical inference on parameters in $\varepsilon=\left\{\varepsilon_{y}, \varepsilon_{\beta}, \varepsilon_{\theta}\right\}$ by utilizing the Gibbs sampler together with the Metropolis-Hastings algorithm for our established models.

\section{SIMULATION AND BAYESIAN ESTIMATIONS}

Basing on the above presented, to illustrate the method proposed we carried out the simulation study, let $n=200, m=5$, we generate each of 50 replicated data sets $\left\{\left(y_{i}, U_{i}, W_{i}, Z_{i}\right): i=1, \ldots, n\right\}$ from a Poisson distribution with the probability density

$$
p\left(y_{i} \mid \gamma_{i}\right)=\frac{\gamma_{i}^{y_{i}} \exp \left\{-\gamma_{i}\right\}}{y_{i} !} .
$$

Where $\lambda_{i}=\log \left(\gamma_{i}\right)=Z_{i}^{T} \alpha_{z}+U_{i}^{T} \alpha_{V}=X_{i}^{T} \alpha$. Let $U_{i} \sim N\left(0,0.25 I_{3}\right)$ and $Z_{1 i}$ and $Z_{2 i}$ in $Z_{i}$ were generated via Equation (4). In this case, $\psi$ relating to Equation (1) is a constant. The true values of $\alpha_{z}, \alpha_{V}, \beta_{k}$ and $\sigma_{z}^{2} \quad$ were taken to be $\quad \alpha_{z}=(0.4,-0.3)^{T}, \alpha_{v}=(0.3,0.3,0.4)^{T}$, $\beta_{k}=(0.2,0.2,0.2,0.5)^{T}$ for $k=1$ and 2 , and $\sigma_{z}^{2}=1$, respectively. To test the effectiveness of using the TCDP prior to approximate distributions of MEs $\theta_{i j}=\left(\theta_{i j 1}, \theta_{i j 2}\right)^{T}$, we set the following distributional simulation for $\theta_{i j k}$.

Simulation 1: We specify that $\theta_{i j k}=0.25\left(\theta_{i j 1}-1\right)+0.75\left(\theta_{i j 2}-0.4\right)$ be from $\theta_{i j 1} \sim \Gamma(1,1)$ and $\theta_{i j 2} \sim \operatorname{Beta}(2,3)$.

Simulation 2: We suppose that $\theta_{i j k}=0.25\left(\theta_{i j 1}-0.5\right)+0.75\left(\theta_{i j 2}-0.25\right) \quad$ be from $\theta_{i j 1} \sim \operatorname{Beta}(4,4)$ and $\theta_{i j 2} \sim \operatorname{Beta}(1,3)$.

We considered the following three types of priors for $\alpha$ and $\beta_{k}$ to investigate sensitivity of Bayesian estimates to prior inputs.

Type A. The hyperparameters corresponding to the priors of $\alpha$ and $\beta_{k}$ were taken to be $\alpha^{0}=(0.4,-0.3,0.3,0.3,0.4)^{T} \quad, \quad H_{\alpha}^{0}=0.25 I_{5}$, $\beta_{k}^{0}=(0.2,0.2,0.2,0.5)^{T}$ and $H_{\beta k}^{0}=0.25 I_{4}$. This can be regarded as a situation with good prior information.

Type B. The hyperparameters corresponding to the priors of $\alpha$ and $\beta_{k}$ were taken to be $\quad \alpha^{0}=1.5 \times(0.4,-0.3,0.3,0.3,0.4)^{T}$, $\beta_{k}^{0}=1.5 \times(0.2,0.2,0.2,0.5)^{T} \quad H_{\alpha}^{0}=0.75 I_{5} \quad$ and $H_{\beta k}^{0}=0.75 I_{4}$. This can be regarded as a situation with inaccurate prior information.

Type C. The hyperparameters corresponding to the priors of $\alpha$ and $\beta_{k}$ were taken to be $\alpha^{0}=0 \times(0.4,-0.3,0.3,0.3,0.4)^{T}$ $\beta_{k}^{0}=0 \times(0.2,0.2,0.2,0.5)^{T} \quad, \quad H_{\alpha}^{0}=10 I_{5} \quad$ and $H_{\beta k}^{0}=10 I_{4}$. This can be regarded as a situation with noninformative prior information.

The simulation results are summaried in Table 1 and Table 2, after 5000 burn-in iterations 5000 observations for each of the generated 50 data sets were collected to evaluate Bayesian estimates via Markov chain Monte Carol (MCMC) samples from the full data posterior distribution. Results under assumption together with three types of prior inputs 
were presented in Table 1-2, in Table 1-2 where 'Bias' was the absolute difference between the true value and the mean of the estimates based on 50 replications and 'RMS' was the root mean square between the estimates based on 50 replications and its true value.

TABLE I. PARAMETER ESTIMATES IN THE First SimUlation

\begin{tabular}{cccccccc}
\hline Parameter & $\begin{array}{c}\text { True } \\
\text { value }\end{array}$ & $\begin{array}{c}\text { Type } \\
\text { Bias }\end{array}$ & $\begin{array}{c}\text { A } \\
\text { RMS }\end{array}$ & $\begin{array}{c}\text { Type } \\
\text { Bias }\end{array}$ & $\begin{array}{c}\text { B } \\
\text { RMS }\end{array}$ & $\begin{array}{c}\text { Type } \\
\text { Bias }\end{array}$ & $\begin{array}{c}\text { C } \\
\text { RMS }\end{array}$ \\
\hline$\beta_{10}$ & 0.2 & 0.0004 & 0.0729 & 0.0146 & 0.0772 & 0.0065 & 0.0669 \\
$\beta_{11}$ & 0.2 & 0.0394 & 0.1231 & 0.0074 & 0.1436 & 0.0044 & 0.1558 \\
$\beta_{12}$ & 0.2 & 0.0063 & 0.1459 & 0.0435 & 0.1456 & 0.0432 & 0.1662 \\
$\beta_{13}$ & 0.5 & 0.0045 & 0.1097 & 0.0228 & 0.1385 & 0.0078 & 0.1645 \\
$\beta_{20}$ & 0.2 & 0.0119 & 0.0758 & 0.0140 & 0.0649 & 0.0005 & 0.0635 \\
$\beta_{21}$ & 0.2 & 0.0113 & 0.1272 & 0.0007 & 0.1164 & 0.0207 & 0.1626 \\
$\beta_{22}$ & 0.2 & 0.0062 & 0.1222 & 0.0163 & 0.1240 & 0.0418 & 0.1571 \\
$\beta_{23}$ & 0.5 & 0.0148 & 0.1358 & 0.0481 & 0.1577 & 0.0154 & 0.1681 \\
$\alpha_{0}$ & 0.4 & 0.0037 & 0.0558 & 0.0221 & 0.0698 & 0.0101 & 0.0526 \\
$\alpha_{1}$ & -0.3 & 0.0023 & 0.0695 & 0.0086 & 0.0601 & 0.0130 & 0.0621 \\
$\alpha_{2}$ & 0.3 & 0.0247 & 0.1193 & 0.0584 & 0.1380 & 0.0163 & 0.1150 \\
$\alpha_{3}$ & 0.3 & 0.0206 & 0.1229 & 0.0184 & 0.1348 & 0.0260 & 0.1383 \\
$\alpha_{4}$ & 0.4 & 0.0191 & 0.1361 & 0.0018 & 0.1349 & 0.0233 & 0.1337 \\
$\sigma_{z}^{2}$ & 1 & 0.0065 & 0.0707 & 0.0011 & 0.0574 & 0.0038 & 0.0696 \\
\hline
\end{tabular}

TABLE II. PARAMETER ESTIMATES IN THE SECOND SimUlation

\begin{tabular}{cccccccc}
\hline Parameter & $\begin{array}{c}\text { True } \\
\text { value }\end{array}$ & $\begin{array}{c}\text { Type } \\
\text { Bias }\end{array}$ & $\begin{array}{c}\text { A } \\
\text { RMS }\end{array}$ & $\begin{array}{c}\text { Type } \\
\text { Bias }\end{array}$ & $\begin{array}{c}\text { B } \\
\text { RMS }\end{array}$ & $\begin{array}{c}\text { Type } \\
\text { Bias }\end{array}$ & $\begin{array}{c}\text { C } \\
\text { RMS }\end{array}$ \\
\hline$\beta_{10}$ & 0.2 & 0.0197 & 0.0755 & 0.0137 & 0.0771 & 0.0105 & 0.0683 \\
$\beta_{11}$ & 0.2 & 0.0050 & 0.1297 & 0.0071 & 0.1599 & 0.0137 & 0.1406 \\
$\beta_{12}$ & 0.2 & 0.0194 & 0.1002 & 0.0004 & 0.1414 & 0.0210 & 0.1647 \\
$\beta_{13}$ & 0.5 & 0.0009 & 0.1184 & 0.0206 & 0.1506 & 0.0440 & 0.1444 \\
$\beta_{20}$ & 0.2 & 0.0053 & 0.0820 & 0.0204 & 0.0725 & 0.0115 & 0.0774 \\
$\beta_{21}$ & 0.2 & 0.0001 & 0.1219 & 0.0320 & 0.1446 & 0.0152 & 0.1570 \\
$\beta_{22}$ & 0.2 & 0.0279 & 0.1230 & 0.0113 & 0.1195 & 0.0008 & 0.1044 \\
$\beta_{23}$ & 0.5 & 0.0073 & 0.1220 & 0.0286 & 0.1347 & 0.0139 & 0.1340 \\
$\alpha_{0}$ & 0.4 & 0.0054 & 0.0411 & 0.0121 & 0.0502 & 0.0034 & 0.0544 \\
$\alpha_{1}$ & -0.3 & 0.0053 & 0.0603 & 0.0131 & 0.0662 & 0.0031 & 0.0752 \\
$\alpha_{2}$ & 0.3 & 0.0248 & 0.1141 & 0.0333 & 0.1451 & 0.0214 & 0.1110 \\
$\alpha_{3}$ & 0.3 & 0.0106 & 0.1136 & 0.0336 & 0.1233 & 0.0218 & 0.1573 \\
$\alpha_{4}$ & 0.4 & 0.0106 & 0.1117 & 0.0203 & 0.1172 & 0.0020 & 0.1281 \\
$\sigma_{z}^{2}$ & 1 & 0.0086 & 0.0615 & 0.0032 & 0.0698 & 0.0039 & 0.0668 \\
\hline
\end{tabular}




\section{CONCLUSION}

From Tables 1-2 we can see that (i) Bayesian estimates were reasonably accurate regardless of distributional assumptions of $\theta_{i j k}$ and prior inputs of unknown parameters because their Bias values were less than 0.10 and their RMS values were less than 0.20; (ii) the Dirichlet prior can be general enough to capture characteristics of various distributional assumptions for MEs; (iii) the results is conducted to illustrate the distribution of $\theta_{i j k}$ can be estimated well by our proposed method.

\section{ACKNOWLEDGEMENT}

This work is supported by the introduction of talent project of Huizhou University of China (2015JB018).

\section{REFERENCES}

[1] X. H. Lin and R .J. Carroll , Semiparametric regression for clustered data using generalized estimating equations, J. Am. Statist. Assoc. 96 (2001) 1045-1056.

[2] W. A . Fuller, Measurement error models, John Wiley, New York, 1987.
[3] R. J. Carroll, D. Ruppert, L. A. Stefanski and C. M. Crainiceanu, Measurement Error Models in Nonlinear Models, 2nded, Chapman and Hall ,New York, 2006.

[4] N. S. Wang, X. H. Lin, R. G. Gutierrez and R. J. Carroll, Bias Analysis and SIMEX Approach in Generalized Linear Mixed Measurement Error Models, J. Am. Ștatist. Assoc. 93 (1998) 249-261.

[5] H. Liang, W. H $\vec{a}$ rdel and R. J. Carroll, Estimation in a Semiparametric Partially Linear error-in-Variables Models, The annals of Statistics. 27 (1999) 1519-1535.

[6] Y. Y. Ma and R. J. Carroll, Locally efficient Estimators for Semiparametric Models With Measurement Error, J. Am. Statist. Assoc. 101 (2006) 1465-1474.

[7] H. Liang, S. J. Wang and R. J. Carroll, Partially Linear Models With Missing Response Variables and Error-prone Covariates, Biometrika. 94 (2007) 185-198.

[8] W. Q. Pan, D. L. Zeng and X. H. Lin, Estimation in semiparametric Transition Measurement error Models for longitudinal data, Biometrica. 65(2008) 728-736.

[9] S. Geman and D. Geman, Stochastic relaxation, Gibbs distribution, and the Bayesian restoration of images, IEEE Transactions on Pattern Analysis and Machine Intelligence 6 (1984) 721-741.

[10] V. H. Lachos, V. G. Cancho and R. Aoki, Bayesian analysis of skew-t multivariate null intercept measurement error model, Stat Pap. 51 (2010) 531-545. 